Improved AIRS/AMSU Surface and Atmospheric Soundings Under Partial Cloud Cover Using an AIRS Only Cloud Clearing Approach

\author{
Joel Susskind \\ NASA Goddard Space Flight Center, Greenbelt, MD, USA 20771 \\ Popular Summary
}

AIRS was launched on EOS Aqua on May 4, 2002, together with AMSU-A and HSB, to form a next generation polar orbiting infrared and microwave atmospheric sounding system. This paper describes the latest scientific advances made in the AIRS Science Team Version 5.0 retrieval algorithm. Starting in early 2007, the Goddard DAAC will use this algorithm to analyze near real time AIRS/AMSU observations. These products are then made available to the scientific community for research purposes. The products include twice daily measurements of the Earth's three dimensional global temperature, water vapor, and ozone distribution as well as cloud cover. In addition, accurate twice daily measurements of the earth's land and ocean temperatures are derived and reported. Scientists use this important set of observations for two major uses. They provide important information for climate studies of global and regional variability and trends of different aspects of the earth's atmosphere. They also provide information for researchers to improve the skill of weather forecasting. A very important new product of the AIRS Version 5 algorithm is accurate case-by-case error estimates of the retrieved products. This heightens their utility for use in both weather and climate applications. These error estimates are also used directly for quality control of the retrieved products. 


\title{
Improved AIRS/AMSU Surface and Atmospheric Soundings under Partial Cloud Cover using an AIRS Only Cloud Clearing Approach
}

\author{
Dr. Joel Susskind \\ Laboratory for Atmospheres \\ NASA Goddard Space Flight Center \\ Mail Code 613.5 \\ Greenbelt, MD 20771 \\ Joel.Susskind-1@nasa.gov
}

\begin{abstract}
The AIRS Version-5 cloud clearing and sounding methodology will be described briefly and results will be shown. These results demonstrate the importance of having sounding channels with very low noise extending to $2400 \mathrm{~cm}^{-1}$.

2007 Optical Society of America

OCIS codes: (300.2140) Emission; (300.6340) Spectroscopy, Infrared
\end{abstract}

\section{Introduction}

The AIRS Science Team Version 5.0 retrieval algorithm will become operational at the Goddard DAAC in early 2007 in the near real-time analysis of AIRS/AMSU sounding data. This algorithm contains many significant theoretical advances over the AIRS Science Team Version 4.0 retrieval algorithm used previously. Two very significant developments are: 1) the development and implementation of a very accurate Radiative Transfer Algorithm (RTA) which allows for accurate treatment of non-Local Thermodynamic Equilibrium (non-LTE) effects on shortwave sounding channels; and 2) the development of methodology to obtain very accurate case by case product error estimates which are in turn used for quality control. These theoretical improvements taken together enabled a new methodology to be developed which further improves soundings in partially cloudy conditions, without the use of microwave observations in the cloud clearing step as has been done previously. In this methodology, longwave $\mathrm{CO}_{2}$ channel observations in the spectral region $710 \mathrm{~cm}^{-1}$ to $750 \mathrm{~cm}^{-1}$ as well as 11 micron window channels, are used exclusively for cloud clearing purposes, while shortwave $\mathrm{CO}_{2}$ channels in the spectral region $2195 \mathrm{~cm}^{-1}$ to $2395 \mathrm{~cm}^{-1}$ and 4 micron window channels, are used for surface and atmospheric temperature sounding purposes.

\section{Steps in the AIRS only Cloud Clearing Approach}

AIRS retrievals use cloud cleared radiances for channel $\mathrm{i}, \hat{\mathrm{R}}_{\mathrm{j}}{ }^{\mathrm{n}}$, which represent the radiances which "would have been observed if the scene were cloud free." The AIRS only cloud clearing retrieval system is very similar to that used previously. Cloud clearing uses AIRS observations in multiple adjacent fields of view, in conjunction with an $n^{\text {th }}$ estimate of the surface and atmospheric state, $X^{n}$. A more accurate estimate $X^{n}$ generally results in better values of $\hat{R}_{i}{ }^{n}$.

The AIRS cloud clearing and retrieval algorithm requested uses the following steps.

1) Cloudy regression produces $X^{1}$.

2) $\hat{R}_{i}{ }^{1}$ computed using $X^{1}$.

3) Generate $X^{\text {reg }}$ using $\hat{R}_{i}{ }^{1}, X^{\text {reg }}=X^{2}$.

4) $\hat{R}_{i}{ }^{2}$ computed using $X^{2}$.

5) Physical retrieval gives $X^{3}$. Uses $\hat{R}_{i}{ }^{2}$, starts with $X^{2}$.

6) $\hat{R}_{i}^{3}$ computed using $X^{3}$. 
7) Physical retrieval-uses $\hat{\mathrm{R}}_{\mathrm{i}}{ }^{3}$, gives solution $X^{4}$.

Cloudy regression (Step 1) and regression (Step 3) use all AIRS channels. Physical retrieval and cloud clearing steps use selected AIRS channels.

\section{Channels used in the AIRS cloud clearing and physical retrieval}

The channels used in the AIRS cloud clearing and physical retrieval steps are shown in Figure 1. Cloud clearing uses only long wave channels between $655 \mathrm{~cm}^{-1}$ and $812 \mathrm{~cm}^{-1} .15 \mu \mathrm{m}$ channels between $664 \mathrm{~cm}^{-1}$ and $713 \mathrm{~cm}^{-1}$ are used to sound the stratosphere and upper troposphere. The majority of information in the physical temperature profile retrieval step is obtained from channels between $2360 \mathrm{~cm}^{-1}$ and $2395 \mathrm{~cm}^{-1}$. In addition, surface skin temperature, as well as SW surface spectral emissivity and shortwave surface bidirectional reflectance of solar radiation are, as solved for using only shortwave window channels. This follows the approach of Chahine et al., which showed that it is preferable to use only long wave channels for cloud clearing and shortwave channels for sounding purposes so as to be least affected by errors in the state $\mathrm{X}^{\mathrm{n}}$ used to generate the cloud cleared radiances.
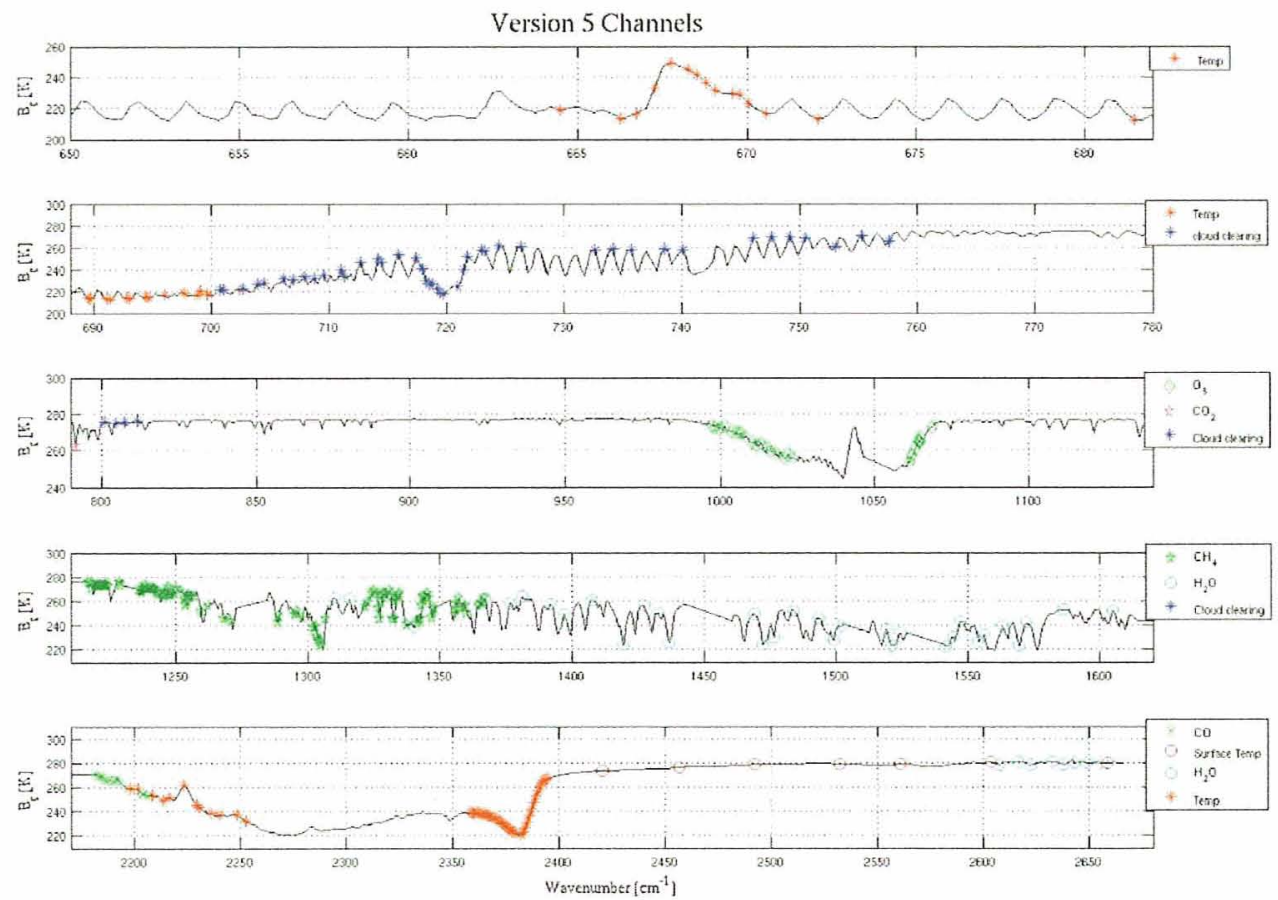

Figure 1

\section{Results}

Figure 2 shows RMS errors vs. ECMWF "truth" of global nighttime and daytime retrievals using the AIRS only retrieval system, both with and without internal quality control (QC). Also shown are the errors of the intermediate steps: cloudy regression and regression. The QC involves residuals of various steps in the cloud clearing and physical retrieval system. It is particularly influenced by the difference of observed (cloud cleared) radiances and those computed from the solution in the critical spectral range $2360 \mathrm{~cm}^{-1}$ to $2395 \mathrm{~cm}^{-1}$.

The cloudy regression without QC (dashed blue line) is based on observed radiances only and is influenced primarily by observations in the $\mathrm{CO}_{2} 15 \mu \mathrm{m}$ spectral band. Very large errors exist in this ensemble of 
cases. Use of internally generated quality control, heavily based on the shortwave $\mathrm{CO}_{2}$ sounding channels (solid blue line) improves these results considerably. The red lines, representative of regression, with and without QC, use both physics (for cloud clearing) and statistics. Both of these depend mostly on the $15 \mu \mathrm{m}$ $\mathrm{CO}_{2}$ channels. The second step improves significantly over the first step, especially after applying quality control. The physical retrieval (black lines) is based almost exclusively on the $4.2 \mu \mathrm{m} \mathrm{CO} \mathrm{C}_{2}$ channels and further improves results significantly compared to the regression based retrievals, especially after internally generated QC. This finding holds under both daytime and nighttime conditions.
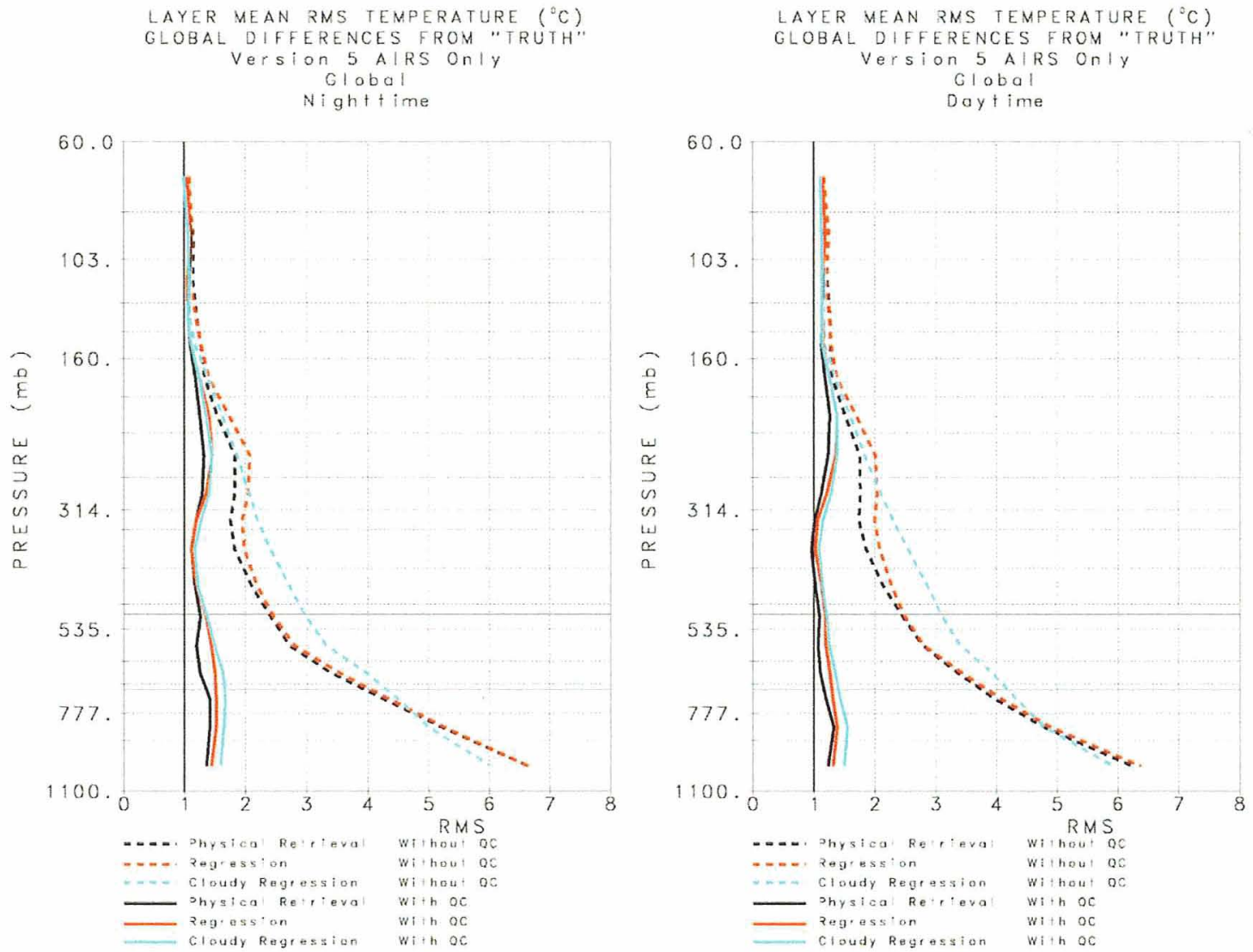

Figure 2

These results demonstrate the importance of having sounding channels in the spectral range $2360 \mathrm{~cm}^{-1}$ to $2400 \mathrm{~cm}^{-1}$, with very low noise (NEDT@250K $<0.3 \mathrm{~K}$ ), on AIRS as well as on future high spectral resolution IR sounders. 